\title{
Variabilité clonale de la race polonica du mélèze d'Europe pour la résistance à Lachnellula willkommii
}

\author{
Luc E. Pâques ${ }^{\mathrm{a}^{*}}$, Giberte Sylvestre-Guinot ${ }^{\mathrm{b}}$, Claude Delatour ${ }^{\mathrm{b}}$ \\ a Station d'amélioration des arbres forestiers, Inra, centre d'Orléans, 45160 Olivet, France \\ ${ }^{b}$ Laboratoire de pathologie forestière, Inra, centre de Nancy, 54280 Champenoux, France
}

(Reçu le 9 janvier 1998 ; accepté le 19 août 1998)

\begin{abstract}
Genetic variation among clones of Larix decidua polonica for resistance to Lachnellula willkommii. Forty clones of European larch selected from four native stands in the Blizyn region (central Poland) were tested for resistance to Lachnellulla willkommii. In spring 1991, artificial inoculation (mycelium on sorghum straw inserted into a hole drilled in twigs) was conducted in three French sites (Orléans, Peyrat-le-Château and Cendrieux). Each clone represented by between 3 to 1511 -year-old ramets received between 20 to 40 inoculations. The development of larch canker symptoms (bark necrosis, pathogenic resins, fructification) was observed for three growing seasons. The overall infection rate ( $T i$, defined as the proportion of inoculation points showing at least one of the symptoms) varied from 10 to $30 \%$ according to the site. This confirms the rather weak to moderate susceptibility to canker of the polonica race and reveals a strong site effect on the infection success and further development. A broad clonal variability is shown (worthy of mention is the absence of an immune clone) together with a sufficiently high level of genetic control $\left(\mathrm{h}^{2}{ }_{\mathrm{bs}}\right.$ : $0.53-0.66$ for $T i$ ) for the selection of clones for resistance to be conducted efficiently. Nevertheless, two main factors somewhat limit the scope of this study $: i$ ) the instability of ranking of clones over sites and $i i)$ a large within-clone variability. Progress in the experimental use of this artificial inoculation technique is expected from a better understanding of the host-pathogen interaction process. In particular are suggested $: i$ ) the definition of optimal ecological conditions for the pathogen expression (for the choice of the experimental site) and ii) a limitation of undesirable experimental noises - e.g. it is hypothesised that the vigour of the inoculated twig can influence the infection success, in which case the standardisation of twig size is necessary. (@ Inra/Elsevier, Paris.)
\end{abstract}

\section{Lachnellula willkommi / Larix decidua polonica / inoculation / clonal variability / resistance / canker}

Résumé - Quarante clones de Larix decidua originaires du centre de la Pologne ont été inoculés dans trois sites français (Orléans, Peyrat-le-Chateau, Cendrieux) avec un isolat de Lachnellula willkommii au printemps 1991. Chaque clone représenté par 3 à 15 ramets âgés de 11 ans, a reçu 20 à 40 inoculations. Les symptômes ont été suivis pendant trois saisons de végétation. Le taux général d'infection obtenu a varié de 10 à $30 \%$ selon les sites, confirmant une sensibilité faible à modérée de la race « polonica " étudiée et révélant un effet marqué du site sur la réussite et le déroulement de l'infection. Une forte variabilité clonale (à noter l'absence de clone immun) est mise en évidence ainsi qu'un contrôle génétique non négligeable de la résistance au niveau des moyennes clonales, mais le classement des clones varie selon les sites. La variabilité résiduelle (intraclonale) forte, due aux effets du milieu reste un obstacle à la réalisation de tests à portée générale. Des progrès sont attendus d'une meilleure connaissance de l'intéraction hôte-parasite, en particulier par la définition des conditions optimales d'expression de la maladie (en particulier pour le choix du site expérimental) et d'autre part par une réduction des bruits de fond liés à l'expérimentation (par exemple homogénéisation de la taille des organes inoculés). (O Inra/Elsevier, Paris.)

Lachnellula willkommii / Larix decidua polonica / inoculation / variabilité clonale / résistance / chancre

* Correspondance et tirés à part

paques@orleans.Inra.fr 


\section{INTRODUCTION}

L'extension du mélèze en-dehors de son aire naturelle (Alpes) a été largement compromise en Europe occidentale et en France en particulier, par le transfert de provenances alpines mal adaptées pour les reboisements de plaine. Dans ces conditions écologiques, il est souvent victime du chancre provoqué par Lachnellula willkommii (Hartig) Dennis, une des maladies les plus dommageables pour le mélèze [21].

Les études conduites en Europe depuis 1944 sur la variabilité naturelle du mélèze d'Europe ( $1^{\text {re }}$ et $2^{\text {e }}$ expériences internationales IUFRO) ont confirmé que les populations alpines sont plus sensibles à la maladie que celles d'Europe centrale : Sudètes ( $L$. sudetica) et Centre Pologne (L. polonica) [16].

Outre les recommandations faites aux reboiseurs en faveur des provenances des Sudètes et du Centre Pologne, ces résultats ont incité les améliorateurs à s'intéresser préférentiellement à ces deux populations par ailleurs vigoureuses et de grande plasticité. Le programme d'amélioration génétique du mélèze conduit en France par l'Inra gère plusieurs centaines de clones de ces deux races et la résistance au chancre y est un des critères majeurs de sélection. Ce choix résulte du fait qu'aucune population n'apparaît totalement résistante : cela a été observé aussi bien en conditions naturelles dans plusieurs dispositifs expérimentaux européens [16] qu'à la suite d'inoculations artificielles [20].

Le développement d'une méthode d'inoculation du parasite et la définition de ses conditions d'utilisation [19] nous ont incités à envisager de tester systématiquement par voie d'inoculation tous les clones des populations d'amélioration du mélèze d'Europe. Ces clones installés en verger à graines sont destinés à produire des variétés synthétiques adaptées aux reboisements de basse et moyenne altitude.

L'objectif principal du présent travail a donc été de déterminer la sensibilité relative à la maladie, de différents clones du mélèze d'Europe polonica, réputé le plus résistant. Cette connaissance doit servir de base à l'élimination sélective des clones les moins intéressants du point de vue de la résistance. Directement lié au précédent, un second objectif, a été d'estimer la stabilité du comportement de l'hôte vis-à-vis du parasite. En effet, de nombreux auteurs ont rapporté que le milieu écologique et la sylviculture exercent une influence marquée sur l'expression de la maladie $[3,8,16,22,23]$. Pour tenir compte de cette influence, nous avons étudié comparativement le comportement du matériel végétal dans trois sites expérimentaux écologiquement très différents.

\section{MATÉRIEL ET MÉTHODES}

\subsection{Matériel végétal}

Quarante clones de mélèze d'Europe (Larix decidua Mill.) originaires des collines de Ste-Croix (entre Kielce et Radom dans le Centre de la Pologne) ont été utilisés, dont 32 sont communs aux trois sites expérimentaux. Il s'agit de 40 arbres «plus » sélectionnés phénotypiquement par l'Institut de recherches forestières de Varsovie (IBL) pour leur forte croissance et la bonne forme de leur tige. Ces arbres proviennent de quatre peuplements voisins, réputés autochtones: Jastrzebia (Blizyn) : clones 243 à 253, Swinia Gora (Blizyn) : clones 254 à 268, Wilczy Bor (Skarzysko Blizyn) : clones 269 à 270 et Jastrzebia (Skarzysko Blizyn) : clones 271 à 282. Ces clones constituent la base de la population d'amélioration (race 'polonica') gérée par la Station d'amélioration des arbres forestiers de l'Inra.

Le matériel greffé à l'Inra d'Orléans au printemps 1979 a été installé à partir de 1980 en collections clonales sur trois sites : Orléans (Loiret), Peyrat-le-Château (Haute Vienne) et Cendrieux (Dordogne). Chaque clone y est représenté par 3 à 15 ramets. Les conditions écologiques très contrastées de ces trois sites faisait de ces collections un matériel intéressant pour étudier les intéractions génotype-site (respectivement, altitude : 107, 570 et $200 \mathrm{~m}$; précipitation annuelle moyenne : 620 , 1260 et $880 \mathrm{~mm}$; température annuelle moyenne, minimum-maximum : $6,2-14,9,3,4-14,6$ et $5,9-17,3^{\circ} \mathrm{C}$ ).

\subsection{Inoculum et mode d'inoculation}

\subsubsection{Inoculum}

La souche de $L$. willkommii utilisée provient d'un chancre naturel de $L$. decidua de l'Arboretum d'Amance (54). Depuis sa première obtention en 1987 , elle a été maintenue sur mélèze vivant par inoculations successives; elle a été utilisée dans nos expériences antérieures $[18,19]$. Dans la présente expérience, l'isolat ré-obtenu était âgé d'environ 3 mois au moment des inoculations.

\subsubsection{Les inoculations.}

Les inoculations ont été réalisées fin avril 1991. Elles ont été effectuées sur des rameaux latéraux avec un inoculum mycélien (paille de sorgho) appliqué sur une blessure (perforation radiale de $1 \mathrm{~mm}$ de diamètre) selon la méthode décrite antérieurement [19] mais les blessures ont été pratiquées sans désinfection préalable des rameaux. Les rameaux inoculés étaient tous âgés de 
2 ans et d'un diamètre toujours supérieur à $3 \mathrm{~mm}$. Chaque rameau inoculé a reçu également une blessure témoin située en position distale par rapport à la blessure inoculée, distante de 7-10 cm de celle-ci. Du fait du grand nombre de rameaux inoculés, leur diamètre initial n'a pas été noté et les points d'inoculation n'ont pas été répertoriés individuellement (1560 blessures inoculées à Orléans, 1520 à Peyrat et 760 à Cendrieux, comprenant un nombre moyen de répétitions par clone de 42,40 et 22 respectivement).

\subsection{Observations}

Tous les points inoculés ainsi que les témoins ont été observés au bout de 15 à 18 ou 27 mois (soit août 92 et juillet 93 à Orléans, octobre 92 à Peyrat, juillet 92 et août 93 à Cendrieux). La seconde notation n'a pas été faite à Peyrat, l'essai y ayant été interrompu après deux saisons de végétation (oct. 92, 18 mois). En effet, l'on craignait une diffusion du pathogène dans la pépinière de production à partir des apothécies déja apparues chez les infections en cours. A chaque notation, le diamètre des rameaux inoculés a été mesuré; la partie mesurée a été choisie en position basale la plus proche possible de l'inoculation, mais non déformée par le chancre.

Les critères pris en compte pour évaluer l'infection (symptômes : présence/absence) ont été les mêmes que ceux définis antérieurement par Sylvestre-Guinot et Delatour [20] à savoir : pour la présence du champignon : (1) les fructifications imparfaites (glomérules conidifères) et les fructifications parfaites (apothécies); pour les manifestations pathologiques : (2) la nécrose de l'écorce (dépression visible extérieurement) et (3) l'exsudation pathologique de résine (résine fraîche).

L'importance de la nécrose d'écorce a été aussi prise en compte par mesure de la longueur totale de la dépression et par l'estimation (par quart) de son extension latérale (ceinturation du rameau).

Nous définissons le taux de présence d'un critère donné comme sa fréquence parmi les inoculations lues dont le nombre a varié d'une notation à l'autre du fait de la disparition accidentelle de certaines d'entre elles. De façon plus globale, les inoculations jugées réussies ont été celles chez qui au moins un des critères était présent; leur fréquence est exprimée par le taux d'infection (Ti). Compte tenu des résultats antérieurs obtenus en matière de jugement des inoculations [20], il n'a pas été pratiqué de réisolements.

\subsection{Analyses statistiques et génétiques}

Pour l'étude comparative du déroulement de la maladie entre les trois sites expérimentaux, c'est tous ramets confondus que l'évolution des divers symptômes et du taux d'infection a été considérée.

Pour l'étude des performances clonales, les analyses ont en revanche été réalisées à partir des fréquences moyennes par ramet. S'agissant de pourcentages, nous avons régularisé les effectifs en éliminant les résultats qui portaient sur un nombre trop faible de points lus par ramet (inférieur à 6). L'expérience conduite selon un schéma considéré comme complètement aléatoire a été analysée par analyse de variance en considérant seul le facteur clone (facteur aléatoire).

L'importance de la ceinturation, la longueur de la dépression ainsi que le diamètre du rameau ont été étudiés au niveau individuel.

Les variables relatives à des fréquences ont été étudiées avec et sans transformation arcsinus. Les résultats étant semblables, seuls les résultats sans transformation sont présentés.

Le coefficient intraclasse $t$ a été calculé pour estimer le niveau de répétabilité des observations :

$$
t=\sigma^{2}{ }^{d\left(\sigma^{2}{ }_{w}+\sigma^{2}\right)}
$$

Il fixe par ailleurs une limite supérieure à l'héritabilité au sens strict.

Faisant l'hypothèse de l'absence d'effet ramet (effet C), l'héritabilité au sens large (au niveau moyenne de clones) a été estimée selon

$$
\mathbf{H} \mathbf{2}_{\mathrm{c}}=\sigma_{\mathrm{G}^{2}} / \sigma^{2}{ }_{\mathrm{PC}}=\sigma^{2}{ }_{\mathrm{C}}\left(\sigma^{2}{ }_{\mathrm{w}} / \boldsymbol{n}+\sigma_{\mathrm{C}}^{2}\right)
$$

avec $\sigma_{\mathrm{G}}^{2}=\sigma_{\mathrm{C}}^{2}=$ variance génétique $=$ variance clonale;

$\sigma_{\mathrm{PC}}^{2}=$ variance phénotypique au niveau moyenne de clones;

$\sigma^{2}{ }_{w}=$ variance résiduelle (variance environnementale);

$n=$ nombre moyen d'observations par clone.

Un intervalle de confiance à $95 \%$ a été calculé suivant [9].

Pour étudier la stabilité des performances clonales dans le temps et dans l'espace, outre le coefficient de rang de Spearman, nous avons utilisé le test statistique non paramétrique de stabilité (S4) de Hühn [7] pour tester l'homéostasie des clones. Après ajustement des valeurs observées aux effets génétiques [13], la variance des rangs des clones classés dans chaque site est calculée et testée par rapport à une distribution $\chi^{2}$. En cas de rejet 
de l'hypothèse nulle (stabilité phénotypique), les clones intéractifs peuvent aussi être mis en évidence.

Pour le site de Cendrieux, des dégâts importants liés au vent (bris de rameaux) ont réduit exagérément le nombre de points observés par ramet dès la première lecture, limitant sérieusement la précision sur ce niveau d'observation. Aussi, aucune analyse de variance ni étude du déterminisme génétique n'ont été entreprises pour ce site. Par contre, le nombre de points observés par clone (>16) a été jugé suffisant pour l'étude du déroulement de la maladie et celle de la stabilité des clones entre sites (tableau I).

\section{RÉSULTATS}

\subsection{Le déroulement de la maladie}

\subsubsection{Le taux d'infection}

Dans aucun des trois sites, les blessures témoins observées à chaque notation, n'ont jamais présenté de symptômes; les blessures se sont cicatrisées rapidement sans produire d'exsudation persistante de résine.

La présence de symptômes, notée vers la fin de la $2^{\mathrm{c}}$ saison de végétation (15-18 mois après l'inoculation), a été observée chez la plupart des clones inoculés : aucun clone n'était indemne à Orléans, quatre l'étaient à Peyrat (soit $10 \%$ des clones) et trois à Cendrieux, différents des précédents (soit $9 \%$ ).

La réussite des inoculations dans leur ensemble, a été cependant relativement modeste, n'atteignant qu'environ $30 \%$ à Orléans et à Cendrieux et seulement $10 \%$ à Peyrat (tableau II). Le taux moyen d'infection par clone a été très variable mais n'a que très rarement atteint ou dépassé $50 \%$. Par ailleurs, aucune évolution importante de la maladie en terme de taux d'infection, n'a été observée entre la seconde et la troisième année de végétation, indiquant une stabilisation de la situation dès la $2^{\mathrm{e}}$ saison de végétation.

\subsubsection{Caractéristiques de la maladie chez les infections jugées réussies}

L'observation des différentes manifestations de la maladie a montré qu'à Orléans comme à Peyrat, celle-ci s'est déroulée de façon classique (tableau III). Dans la totalité des cas où l'infection était réussie il y avait un développement de nécrose dans l'écorce. Cette nécrose était accompagnée très généralement d'une exsudation pathologique de résine. À Peyrat toutefois, la résine fraîche était relativement peu fréquente au moment de l'observation (34\% en Octobre 1992), mais la présence de résine sèche laissait à penser qu'elle avait concerné, plus tôt en saison, $80 \%$ des inoculations jugées réussies. Les fructifications du champignon se sont développées également chez la plupart des inoculations réussies. Lorsque les fructifications étaient présentes, il y avait des apothécies dans $95-100 \%$ des cas à $15-18$ mois dans les deux sites, et à Orléans à 27 mois, dans $62 \%$ des cas. L'apparition des fructifications a cependant été plus précoce à Peyrat (18 mois : $82 \%)$ qu'à Orléans (15 mois : $56 \% ; 27$ mois : $94 \%$ ).

Par comparaison, l'évolution observée à Cendrieux a été assez différente. Une forte manifestation résinique était la règle alors que le développement de nécroses observables était relativement tardif et moins fréquent que dans les deux sites précédents. Il en était de même pour les fructifications, bien que celles-ci comme dans les cas précédents étaient toujours liées à la présence d'une nécrose. Parmi les inoculations réussies qui portaient des fructifications, les apothécies étaient moins fréquentes que dans les deux sites précédents ( $68 \%$ des cas à 15 mois et seulement $12 \%$ à 27 mois).

Les infections réussies ont abouti, au terme de trois saisons de végétation, à la formation de chancres complets (nécrose + résine fraîche + fructifications) dans $71 \%$ des cas à Orléans contre seulement $43 \%$ à Cendrieux. Du fait de l'infection, la mortalité des rameaux inoculés s'est élevée à $10 \%$ à Orléans au bout de 15 mois ( $0 \%$ à Peyrat et à Cendrieux $)$ puis, de façon

Tableau I. Taille de l'échantillon étudié. Nombre moyen de blessures inoculées observées.

\begin{tabular}{lcccc}
\hline & Délai d'observation & Orléans & Peyrat & Cendrieux \\
\hline Par clone & $15-18$ mois & 38,2 & 38,9 & 17,0 \\
& 27 mois & 30,9 & - & 16,6 \\
Par ramet & $15-18$ mois & 13,6 & 8,8 & 4,6 \\
& 27 mois & 11,0 & - & 4,6 \\
\hline
\end{tabular}


Tableau II. Évolution générale de la maladie dans les trois sites.

\begin{tabular}{|c|c|c|c|c|c|c|c|}
\hline & Délai après inoculation & \multicolumn{2}{|c|}{ Orléans } & \multicolumn{2}{|c|}{ Peyrat } & \multicolumn{2}{|c|}{ Cendrieux } \\
\hline Nombre de blessures & 15-18 mois & \multicolumn{2}{|c|}{1527} & \multicolumn{2}{|c|}{1479} & \multicolumn{2}{|c|}{560} \\
\hline \multirow[t]{2}{*}{ inoculées observées } & 27 mois & \multicolumn{2}{|c|}{1237} & \multicolumn{2}{|c|}{-} & \multicolumn{2}{|c|}{547} \\
\hline & & (1) & (2) & (1) & (2) & (1) & (2) \\
\hline \multirow[t]{2}{*}{ Taux d'infection (\%) } & $15-18$ mois & 31 & $8-64$ & 10 & $0-35$ & 29 & $0-53$ \\
\hline & 27 mois & 31 & $6-74$ & - & - & 22 & $0-50$ \\
\hline \multirow[t]{2}{*}{ Nécrose (\%) } & 15-18 mois & 31 & $8-64$ & 10 & $0-35$ & 13 & $0-50$ \\
\hline & 27 mois & 30 & $6-74$ & - & - & 16 & $0-50$ \\
\hline \multirow[t]{2}{*}{ Résine fraîche (\%) } & 15-18 mois & 27 & $5-57$ & 3 & $0-15$ & 28 & $0-50$ \\
\hline & 27 mois & 23 & $5-65$ & - & - & 20 & $0-50$ \\
\hline \multirow[t]{2}{*}{ Fructifications (\%) } & $15-18$ mois & 17 & $3-57$ & 8 & $0-29$ & 8 & $0-27$ \\
\hline & 27 mois & 29 & $6-7 I$ & - & - & 10 & $0-25$ \\
\hline \multirow[t]{2}{*}{ Ceinturation (proportion) } & $15-18$ mois & 58 & $25-100$ & 44 & $10-75$ & 53 & $50-75$ \\
\hline & 27 mois & 77 & $50-100$ & - & - & 53 & $25-100$ \\
\hline \multirow[t]{2}{*}{ Longueur de nécrose (mm) } & $15-18$ mois & 20 & $7-50$ & 21 & $11-45$ & 21 & $12-40$ \\
\hline & 27 mois & 30 & $13-80$ & - & - & 21 & $9-35$ \\
\hline
\end{tabular}

(1) moyenne générale (tous ramets confondus)

(2) amplitude clonale (amplitude individuelle pour ceinturation et longueur de nécrose.)

Tableau III. Caractéristiques des inoculations réussies. Fréquence (échelle en \%) et combinaison des caractères observés.

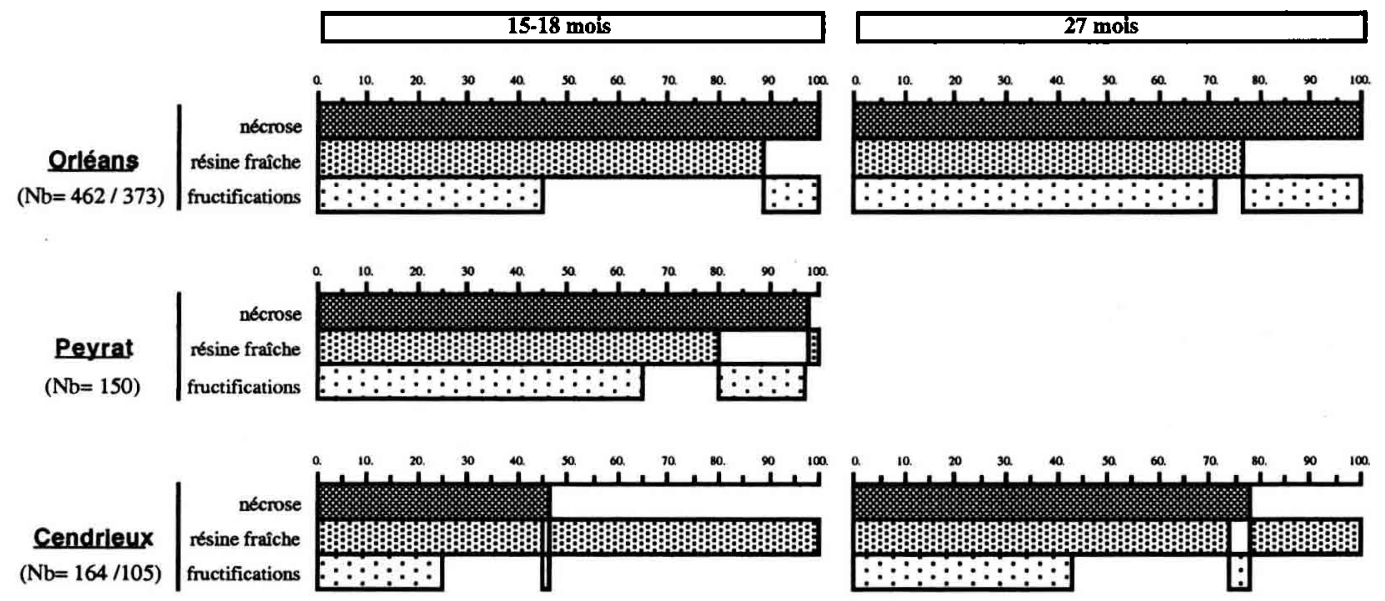

cumulée, à $21 \%$ au bout de 27 mois (moins de $8 \%$ à Cendrieux).

Les mensurations effectuées sur l'ensemble des nécroses dans les trois sites après deux saisons de végétation, ont montré une faible variabilité. La nécrose cein- turait très généralement environ la moitié du rameau inoculé et s'étendait longitudinalement sur une hauteur totale d'une vingtaine de millimètres. Entre la deuxième et la troisième saison de végétation, l'ampleur des nécroses a peu varié à Cendrieux. À Orléans en revanche, les 
nécroses qui couvraient plus de la moitié de la circonférence des rameaux après 15 mois (ceinturation : $57 \%$ ) dépassaient les trois quarts au bout de 27 mois et leur extension était passée de $20 \mathrm{~mm}$ à $30 \mathrm{~mm}$.

Le suivi des manifestations pathologiques montre que par comparaison avec ce qui a été observé à Peyrat et à Orléans, le déroulement de la maladie a eu lieu de façon différente et relativement atypique dans le site de Cendrieux. Notons également pour la suite de l'analyse, que le taux d'infection est totalement redondant avec le taux de nécrose à Orléans et à Peyrat, et qu'il l'est presque avec le taux de résine fraîche à Cendrieux.

\subsection{Performances clonales}

\subsubsection{Variabilité clonale}

Pour tous les symptômes étudiés, mais particulièrement pour le taux d'infection et la fréquence de nécrose, l'amplitude clonale est très large, en particulier sur le site d'Orléans (tableau II). Ceci suggère une forte variabilité génétique. À Peyrat, l'amplitude observée est plus limitée puisque pratiquement réduite de moitié par rapport à Orléans.

Des différences très hautement significatives ( $\alpha=0,001)$ entre clones sont mises en évidence pour tous les caractères à Orléans $\left(\mathrm{F}_{38,67}>2.8^{* * *}\right)$. À Peyrat, les clones diffèrent également entre eux mais avec un taux de signification plus faible qu'à Orléans ( $F_{37}{ }_{32}$ compris entre 1,6 et 2,1 , significatif respectivement au seuil de 5 et $1 \%$ ).

Cependant, une forte variabilité subsiste entre ramets d'un même clone. Pour tous les symptômes (hormis la fréquence de résine fraîche à Peyrat, amplitude $=8,3 \%$ ) et dans chaque site, l'amplitude de réponse entre ramets d'un même clone est toujours supérieure à $18 \%$. Elle dépasse même $25 \%$ pour le taux d'infection et de nécrose à Orléans à 27 mois.

Cela se traduit entre autre par des valeurs du coefficient de corrélation intraclasse $t$, faibles à modérées : 0,12 à 0,43 (tableau $I V$ ) : $t$ est en fait une mesure du niveau de répétabilité d'un symptôme donné puisque les observations sur les divers ramets sont considérées comme des observations multiples d'un même caractère sur un clone donné. La répétabilité est pour tous les caractères plus faible à Peyrat qu'à Orléans et à 15-18 mois moins bonne pour la fréquence de fructifications que pour les autres caractères.
Tableau IV. Répétabilité des observations (coefficient intra-classe).

\begin{tabular}{lccc}
\hline & Sites & Orléans & Peyrat \\
\hline Taux d'infection $(\%)$ & $15-18$ mois & 0,34 & 0,21 \\
& 27 mois & 0,42 & - \\
Nécrose $(\%)$ & $15-18$ mois & 0,34 & 0,21 \\
& 27 mois & 0,42 & - \\
Résine fraîche $(\%)$ & $15-18$ mois & 0,32 & 0,15 \\
& 27 mois & 0,41 & - \\
Fructifications $(\%)$ & $15-18$ mois & 0,22 & 0,12 \\
& 27 mois & 0,43 & - \\
\hline
\end{tabular}

\subsubsection{Contrôle génétique de l'expression de la maladie}

Comme mentionné précédemment, les fréquences moyennes des divers symptômes changent peu entre 15 et 27 mois à Orléans (et à Cendrieux). La liaison entre sensibilité moyenne des clones exprimée entre ces deux périodes peut être considérée comme bonne : les coefficients de rang de Spearman (significativement différents de 0 ) sont de 0,82 pour la fréquence de fructification, 0,64 pour le taux d'infection et la fréquence de nécrose mais seulement de 0,48 pour la fréquence de résine. On peut donc admettre que les mêmes mécanismes génétiques sont en jeu pour les deux périodes.

Compte tenu de la nature du matériel, il est impossible de déterminer le mode précis de transmission génétique des caractères étudiés. Cependant, l'examen des coefficients intraclasse (tableau $I V$ ) donne une indication du niveau de contrôle génétique des symptômes étudiés. Comme indiqué précédemment, celui-ci n'est que modéré à 15-18 mois sur les deux sites d'Orléans et de Peyrat et pour tous les caractères $(<0,34)$. Il s'améliore cependant nettement à 27 mois à Orléans $(>0,41)$.

De manière à pouvoir estimer les possibilités de sélection offertes à l'améliorateur, nous avons calculé une héritabilité estimée sur la base des moyennes clonales (héritabilité au sens large). La sélection porte en effet généralement sur des clones et non sur des individus (ramets), ce qui la rend plus efficace. Ces héritabilités (au niveau moyennes de clones) sont présentées au tableau $V$. Elles montrent un niveau de contrôle génétique moyen $(0,59)$ à 15 mois à Orléans, pour le taux d'infection (et la fréquence de nécrose qui est un critère redondant, cf. tableau III), un peu plus faible pour la fréquence de résine mais surtout pour la fréquence des fructifications. Les résultats sont cohérents sur les deux sites : les héritabilités sont du même ordre de grandeur à 
Tableau V. Héritabilité au niveau des moyennes de clones.

\begin{tabular}{lccc}
\hline $\begin{array}{l}\text { Sites } \\
\text { Délai d'observation }\end{array}$ & $\begin{array}{c}\text { Orléans } \\
15 \text { mois }\end{array}$ & 27 mois & $\begin{array}{c}\text { Peyrat } \\
18 \text { mois }\end{array}$ \\
\hline Taux d'infection & 0,59 & 0,66 & 0,53 \\
& $(0,29-0,78)$ & $(0,40-0,81)$ & $(0,24-0,73)$ \\
Nécrose & 0,59 & 0,66 & 0,53 \\
& $(0,29-0,78)$ & $(0,40-0,81)$ & $(0,24-0,73)$ \\
Résine fraîche & 0,56 & 0,65 & 0,44 \\
& $(0,24-0,76)$ & $(0,39-0,81)$ & $(0,08-0,68)$ \\
Fructifications & 0,43 & 0,67 & 0,37 \\
& $(0,02-0,69)$ & $(0,42-0,82)$ & $(0-0,64)$ \\
\hline
\end{tabular}

(1) intervalle de confiance.

Peyrat. Pour tous les symptômes, les niveaux d'héritabilité augmentent au cours du temps (Orléans : > 0,65 à 27 mois).

Ces héritabilités au sens large ne sont cependant intéressantes à considérer que dans le cas d'une diffusion des clones par voie végétative, ce qui est au moins le cas dans la phase de multiplication par greffage du matériel pour l'installation des vergers à graines. En cas de diffusion après recombinaison sexuée, le coefficient intraclasse $t$ est plus intéressant à considérer. La limite supérieure de l'héritabilité au sens strict est alors fixée par $t$.

\subsubsection{Stabilité clonale inter-sites}

Outre un fort effet du site sur l'expression de la maladie déjà évoqué plus haut, il est intéressant de vérifier l'absence d'interaction entre les 32 clones communs aux trois sites.

Aucune liaison significativement différente de zéro (hormis pour le taux de nécrose entre Orléans et Cendrieux, $\left.r_{S}=0,37^{*}\right)$ n'est mise en évidence $\left(r_{S}<0,31\right.$ ns) entre rangs des clones sur les trois sites, ce qui laisse penser à des changements de rangs importants d'un site à l'autre. Ce résultat est vérifié par le test de stabilité de Hühn [7]. Pour les quatre caractères étudiés, le test montre en effet une inégalité de stabilité entre clones sur les trois sites : $S 4=56,2,60,2,60,0$ et $61,5>\chi^{2} 0,05,32$ ddl pour le taux d'infection, les fréquences de nécrose, de résine fraîche et de fructification. Les clones les plus interactifs sont les clones $250,261,268$ et 275 pour le taux d'infection, 250, 252, 260, 265, 268 et 275 , pour la fréquence de nécrose, $250,257,261,271$ et 275 , pour la fréquence de résine et 244,249,250, 251, 268 et 275 pour la fréquence de fructification.

\section{DISCUSSION}

Les résultats présentés ci-dessus mettent en évidence quatre points principaux.

\subsection{Faible sensibilité du mélèze d'Europe polonica vis-à-vis du $L$. willkommii}

Le taux d'infection mis en évidence dans les trois sites ne dépasse pas $31 \%$. Ce résultat obtenu par inoculation artificielle est à comparer au taux d'infection presque trois fois plus élevé ( $>89 \%$ ) obtenu par Sylvestre et al. [19] chez une provenance de mélèze d'Europe des Alpes françaises avec la même méthode d'inoculation et avec le même isolat du parasite. Le mélèze d'Europe polonica testé dans la présente étude à partir d'un échantillon de clones représentatifs, apparaît donc bien de sensibilité nettement plus faible que le mélèze alpin.

Une conclusion semblable avait déjà été obtenue par Sylvestre et Delatour [20] par recours à une méthode d'inoculation de même type, mais moins bien normalisée (blessure au scalpel inoculée avec du mycelium sur support d'agar) et à partir de six individus seulement. Ils montraient que malgré des taux d'infection semblables, 4 ans après l'inoculation, les trois populations testées (Alpes, Sudètes et Centre Pologne) se comportaient différemment vis-à-vis du pathogène, sans qu'aucun ne soit apparu réfractaire aux inoculations : la provenance alpine à l'opposé de la provenance du Centre Pologne, présentait les perturbations chancreuses les plus importantes.

Ce bon comportement des clones de mélèze d'Europe polonica testés pourrait résulter de leur mode de sélection. En effet, dans le cadre de la sélection phénotypique en forêt (arbres «plus »), tout arbre défectueux, par exemple porteur de chancre, est en principe écarté. Les résultats obtenus ne renseignent donc peut-être pas sur le comportement exact de l'ensemble de la population d'origine, bien que l'incidence de la maladie y soit considérée comme faible ou nulle. Kulej [11] signale par exemple sur un site expérimental des Beskides très propice à la maladie, des taux d'infection inférieurs à $8 \%$ pour les populations de mélèze polonica alors que certaines provenances d'autres régions sont atteintes à plus de $50 \%$.

Ces résultats expérimentaux corroborent également ceux obtenus en situations d'infection naturelle dans les tests de provenances [16] et confirment aussi l'intérêt qui est porté à cette race de mélèze d'Europe dans les programmes d'amélioration génétique. 


\subsection{Variabilité clonale forte et niveau de contrôle génétique modéré}

Parmi la collection des clones testés, aucun ne s'est révélé immun vis-à-vis de la maladie, mais une variabilité très forte entre clones est mise en évidence pour tous les caractères observés. Elle s'exprime d'autant mieux que le site d'expérimentation apparaît plus propice au développement du pathogène, ce qui est à l'évidence le cas à Orléans (6 à $74 \%$ pour le taux d'infection). Parallèlement, le niveau de contrôle génétique est cohérent entre les sites d'Orléans et de Peyrat. Avec des valeurs inférieures à 0,66 au niveau des moyennes de clones, l'héritabilité apparaît modérée pour le taux d'infection, comparée aux niveaux observés dans d'autres tests clonaux pour des caractères comme la rectitude de la tige ou même la hauteur totale [14]. Sans pouvoir être comparé à des résultats similaires sur mélèze, le niveau de contrôle génétique obtenu dans cette étude apparaît équivalent ou supérieur aux niveaux observés pour la résistance à d'autres pathogènes chez d'autres Gymnospermes. Pour la résistance à la rouille courbeuse (Melampsora pinitorqua) chez le pin maritime, Baradat et Desprez-Loustau [1] signalent des niveaux d'héritabilité (au sens strict) compris entre 0,06 et 0,27 que l'on peut comparer à ceux obtenus dans cette étude : $0,21<\mathrm{h} 2_{\mathrm{ss}}<0,42^{\mathrm{l}}$. Dans le cas du couple Endocronartium harknessii $\times$ Pinus contorta des héritabilités de 0,51 au niveau familial et de 0,21 au niveau individuel sont mentionnées par Kojwang [10]. De même pour des clones d'épicéas communs, l'héritabilité ( $=t$ dans notre étude) atteint 0,27 et 0,35 pour deux caractères de sensibilité à Heterobasidium annosum [17].

Sans être donc très élevé, le niveau de contrôle génétique apparaît cependant suffisant pour assurer par sélection clonale, des gains génétiques théoriques appréciables sur chacun des sites : réduction espérée du taux moyen d'infection de $22 \%$ pour un taux de sélection de $5 \%$ et jusqu'à $30 \%$ pour un taux de sélection de $1 \%$.

\subsection{Fort effet du milieu et instabilité clonale inter-sites}

Si la maladie présente sur le mélèze polonica un niveau d'expression plutôt faible, sa manifestation apparaît variable selon le site d'expérimentation à la fois en intensité (du simple au triple pour le taux d'infection

\footnotetext{
${ }^{1}$ le coefficient de corrélation intraclasse représente dans ce cas l'héritabilité au sens strict $\left(\mathrm{h}^{2 \mathrm{ss}}\right)$ si l'on fait l'hypothèse de l'absence d'effet de dominance.
}

moyen entre Orléans et Peyrat) mais aussi dans son déroulement (expression plutôt atypique de la maladie à Cendrieux). Plus grave apparemment dans ses conséquences serait la forte interaction mise en évidence entre le comportement des clones vis-à-vis du parasite et les sites d'expérimentation.

Le problème majeur posé par ces résultats semble donc être lié aux sites. L'influence des conditions écologiques (sol, climat) sur le déroulement des maladies en milieu naturel est connue depuis longtemps en pathologie. Dans le cas par exemple du chancre à Hypoxylon mammatum de Populus tremuloides, il a été montré que certains paramètres chimiques et physiques du sol constituent un ensemble de variables étroitement reliées à la fréquence du chancre en nature [2]. Cependant, aucune expérimentation d'inoculation concernant des maladies chancreuses n'a été rapportée à notre connaissance, utilisant un même isolat d'un parasite donné, appliqué dans différents sites. La comparaison d'un matériel végétal varié se fait classiquement soit par inoculation d'un ou plusieurs isolats en un site donné, soit en installant le matériel végétal dans plusieurs sites, le soumettant à l'infection naturelle. La comparaison des résultats entre ces deux types d'approches est évoquée en Suède par Hanson [6] dans le cas du Gremmeniella abietina.; la plus grande sensibilité de semis de Pinus sylvestris inoculés, comparée à celle du $P$. contorta, est contraire à ce qui est observé en plantations comparatives; à l'appui de cette discordance l'auteur évoque l'absence de stress chez les plants en conditions expérimentales. D'une façon générale, l'instabilité inter-sites de la résistance est considérée comme un indicateur fort d'une interaction génotype $\times$ environnement [4].

Dans notre cas, il serait en particulier intéressant de pouvoir définir des critères de choix du site expérimental qui présenterait les meilleures conditions en vue d'une évaluation optimale et fiable de l'interaction hôte-parasite. L'aspect optimal recherché sous-entend l'obtention d'un taux d'infection suffisant et une expression la plus large possible de la variabilité clonale. Cette condition est d'autant plus critique que le matériel végétal à évaluer est déjà réputé naturellement peu sensible à la maladie comme l'est la population polonica.

À cet égard, le site d'Orléans est clairement apparu comme celui où la maladie se développe le mieux et le plus complètement et où une variabilité clonale s'exprime le plus largement. Par comparaison, le site de Peyrat est apparu nettement moins intéressant du fait d'une trop faible expression de la maladie, Dans le site de Cendrieux, malgré une incidence relativement importante de la maladie, il est à craindre que les résultats obtenus soient peu fiables du fait d'une expression peu typique de la maladie. 
Il n'y a malheureusement pas de raisons évidentes à ce résultat. Pour expliquer ces différences stationnelles, il serait bien sûr utile de pouvoir établir une liaison entre les mécanismes d'installation et de développement du pathogène dans l'hôte et certains critères écologiques pouvant les influencer. Sylvestre-Guinot et Delatour [20] ont par exemple clairement montré que la saison d'inoculation influençait fortement la rapidité d'implantation du pathogène. En conditions naturelles, Bürgi [3] a établi une liaison nette entre la fréquence de tiges chancreuses et le nombre de jours de brouillard sur les sites expérimentaux, mais ceci concerne a priori l'interaction quantité d'inoculum $\times$ portes d'entrée plutôt que la réceptivité proprement dite de l'hôte. Les données dont nous disposons sont insuffisantes pour étudier les facteurs écologiques déterminants d'autant que les effets éventuels de ceux-ci sont confondus avec un traitement cultural des arbres inoculés, différent suivant les sites.

Le faible niveau d'expression de la maladie à Peyrat et le caractère plutôt atypique de la maladie à Cendrieux pourraient par ailleurs être à l'origine des fortes intéractions clones-sites observées, leur donnant éventuellement un poids exagéré dans le cadre de la présente étude.

\subsection{Forte variabilité résiduelle}

Comme indiqué par les coefficients de corrélation intraclasse, la répétabilité de la manifestation des symptômes entre ramets est peu élevée ( $t$ compris à 15-18 mois, entre 0,22 et 0,34 à Orléans et entre 0,12 et 0,21 à Peyrat). Elle est cependant nettement supérieure à celle obtenue dans d'autres études comme celle déjà citée de Baradat et Desprez-Loustau [1] sur la rouille courbeuse du pin maritime $(\mathrm{t}<0,14)$.

Cette répétabilité relativement faible traduit en fait l'existence de 'bruits de fond'non contrôlés qui augmentent exagérément la variance résiduelle (variance environnementale dans notre cas) au détriment de la variance génétique. Deux sources principales de perturbation méritent d'être examinées : la première concerne le schéma expérimental et en particulier la taille de l'échantillon ; la seconde repose sur l'hypothèse d'une influence de la vigueur des organes inoculés sur l'installation et le déroulement de la maladie, déja suggérée par des résultats antérieurs [20].

\subsubsection{Taille de l'échantillon}

Celle-ci concerne d'une part le nombre de ramets utilisés et d'autre part le nombre de points d'inoculation par ramet et donc par clone. Le choix du premier était fixé par le nombre de clones disponibles en plantation alors que celui du second était a priori plus libre.

D'après l'étude de Muranty et al. [12] à partir de données sur Merisier (Prunus avium) et sur mélèze hybride (Larix $\times$ eurolepis), six à huit ramets par clone sont nécessaires pour maximiser la précision d'estimation des valeurs et paramètres génétiques en test clonal. D'autre part, comme le montrent plusieurs auteurs [15], plus le niveau de contrôle génétique (héritabilité) est faible, plus le nombre de ramets doit être important pour évaluer leur valeur. Il est clair maintenant que les nombres de ramets utilisés dans la présente étude ( 3 à 5 ; en réalité le nombre moyen de ramets par clone utilisé en analyse de variance était de 2,7 à Orléans et 4,2 à Peyrat) étaient trop faibles, en particulier compte tenu des niveaux d'héritabilité connus maintenant pour les caractères utilisés pour évaluer la sensibilité au $L$. willkommii.

Un deuxième niveau concerne le nombre d'inoculations par ramet et par clone sur la base duquel sera estimée la fréquence d'apparition des symptômes. Le tableau VI a été construit à partir de [5] pour mettre en évidence le nombre d'observations (donc de points d'inoculation) nécessaires pour d'une part éviter une erreur donnée sur une proportion et d'autre part pour mettre en évidence une différence donnée entre deux proportions. Ainsi, avec les effectifs par clone disponibles à Orléans et à Peyrat (environ 38 points d'inoculation lus), et pour les deux fréquences moyennes extrêmes du taux d'infection ( $10 \%$ et $30 \%$ ) l'erreur commise sur l'estimation des fréquences ne peut être inférieure à 10 et $15 \%$ respectivement. En outre, pour mettre en évidence une différence de l'ordre de $10 \%$ entre les taux d'infection observés chez deux clones, des nombres d'observations de l'ordre de 180 à 400 sont nécessaires; ces nombres sont très supérieurs à ceux que nous avons utilisés dans notre étude et dépassaient largement les possibilités biologiques et techniques de cette expérimentation.

Statistiquement, une augmentation sensible de la proportion moyenne (dans notre cas du taux d'infection moyen $>70 \%$ ) permettrait de travailler avec des effectifs plus faibles. Cependant d'une part, le taux moyen d'infection est dans une large mesure une caractéristique biologique de la population-hôte testée et d'autre part, il n'est pas évident comme indiqué plus haut, de définir aujourd'hui des conditions expérimentales plus favorables à l'expression de la maladie. Dans ces conditions, le seul moyen actuellement disponible pour répondre à cette contrainte d'effectifs serait de travailler sur un nombre de ramets nettement plus élevé. Par exemple, vers l'âge de 5-6 ans, un ramet peut héberger environ 15 points d'inoculation et 15 à 25 ramets seraient donc nécessaires; mais si l'on voulait utiliser des sujets plus jeunes, des ramets de 2-3 ans par exemple qui ne peu- 
Tableau VI. Nombre d'observations nécessaires pour éviter une erreur sur une proportion donnée et nombre d'observations nécessaires pour mettre en évidence une différence entre deux proportions (au seuil de confiance de $5 \%$ et $\mathbf{b}=0,1)$ (d'après [5]).

\begin{tabular}{lcc}
\hline Erreur & Nombre d'observations & Nombre d'observations \\
ou & pour éviter & pour mettre en évidence \\
Différence & l'erreur $d$ & la différence $d$
\end{tabular}

\begin{tabular}{lcccc}
\hline $\boldsymbol{d}$ & \multicolumn{2}{c}{ Proportion moyenne } & \multicolumn{2}{c}{ Proportion moyenne } \\
& $10 \%$ & $30 \%$ & $10 \%$ & $30 \%$ \\
\hline 5 & 144 & 336 & 720 & 1680 \\
$\mathbf{1 0}$ & $\mathbf{3 6}$ & 80 & $\mathbf{1 8 0}$ & $\mathbf{4 0 0}$ \\
$\mathbf{1 5}$ & 16 & $\mathbf{3 7}$ & 80 & 187 \\
20 & 9 & 21 & 45 & 105 \\
25 & 6 & 13 & 29 & 67 \\
\hline
\end{tabular}

vent héberger que trois à quatre inoculations seulement, des effectifs de 45 à 100 ramets seraient nécessaires.

\subsubsection{Influence de la vigueur sur le degré d'infection}

Le fort effet du site sur la maladie, à son installation et au cours de son développement d'une part, et d'autre part le niveau de répétabilité faible à modéré observé entre les réponses des ramets d'un même clone, laissent penser que la vigueur des arbres et des organes inoculés pourraient influencer le degré d'infection, comme cela a déjà été suggéré lors d'études précédentes [20].

Sans pouvoir étudier directement l'influence de la vigueur des rameaux (en terme d'accroissement en diamètre) sur le succès et le développement de l'infection, il nous a semblé intéressant de tester cette hypothèse en mettant en relation infection et diamètre moyen des rameaux.

Comme indiqué au Tableau VII, pour un clone donné, les rameaux infectés ont en moyenne un diamètre significativement plus faible que les rameaux non infectés (test $t$ significatif à $5 \%$ ). Cela est vérifié sur les trois sites et pour les deux périodes de lecture sauf à Cendrieux pour la lecture à 15 mois (pas de différence significative).

Un examen des histogrammes de fréquence (non présentés) indique que les taux d'infection les plus élevés sont assez systématiquement relevés chez les classes des plus petits diamètres disponibles : 4 à $6 \mathrm{~mm}$ ou 6 à $8 \mathrm{~mm}$.

De la même manière, sur des boutures de 4 ans d'épicéa commun, Swedjemark et al. [17] montrent une forte liaison négative $(-0,47$ et- 0,59$)$ entre paramètres de développement d'Heterobasidium annosum et le diamètre de la tige et un index de vigueur. Ils suggèrent ainsi de tenir compte de la vigueur des boutures pour réduire l'erreur expérimentale.

Par ailleurs, une comparaison rapide des résultats entre les trois sites montre assez clairement une relation défavorable entre diamètre moyen des rameaux inoculés et sensibilité à l'infection : Orléans, site où se manifeste la plus grande sensibilité, est caractérisé par le plus faible diamètre moyen; à l'opposé, Peyrat, site où le taux d'infection est le plus faible, présente le plus fort diamètre moyen. Cendrieux est intermédiaire pour les deux caractères. Les différences très marquées de réponse à l'infection entre les sites pourraient donc être partiellement liées à la vigueur des rameaux inoculés.

\section{CONCLUSION}

À travers le comportement des 40 clones testés sur les trois sites expérimentaux utilisés, il apparaît assez clairement que le mélèze d'Europe polonica présente une sensibilité faible à modérée à l'agent du chancre du mélèze, L. willkommii. Cela confirme par voie d'inoculation artificielle, les observations faites aussi bien dans l'aire naturelle que dans les tests de provenances, sous inoculum non contrôlé.

Une forte variabilité clonale ainsi qu'un contrôle génétique non négligeable de la résistance à la maladie sont également mis en évidence. Par sélection, la résistance pourrait donc être augmentée de façon appréciable.

Cependant, l'importante influence du milieu sur l'incidence et le déroulement des infections expérimen- 
Tableau VII. Diamètres moyens des rameaux $\left(e n 1 / 10^{e} \mathrm{~mm}\right)$ et test $t$ entre diamètres moyens des rameaux indemnes et rameaux infectés.

\begin{tabular}{|c|c|c|c|c|c|}
\hline \multirow[b]{2}{*}{ Délai d'observation (mois) } & \multicolumn{2}{|c|}{ Orléans } & \multirow{2}{*}{$\begin{array}{c}\text { Peyrat } \\
18\end{array}$} & \multicolumn{2}{|c|}{ Cendrieux } \\
\hline & 15 & 27 & & 15 & 27 \\
\hline Tous rameaux inoculés & 78,5 & 83,0 & 93,5 & 91,4 & 103,4 \\
\hline Rameaux indemnes & 79,7 & 87,0 & 94,4 & 90,1 & 103,1 \\
\hline Rameaux infectés & 75,8 & 72,2 & 81,0 & 92,0 & 93,7 \\
\hline
\end{tabular}

$*, * *, * * *$ significatif respectivement à 5,1 et $0,1 \%$

tales, probablement par modulation de la réponse de l'hôte, empêche tout classement généralisable des clones entre eux, à partir des résultats obtenus dans la présente étude. Un soin particulier devra être porté au choix des sites expérimentaux, permettant un niveau d'expression correcte de la maladie pour une expression optimale de la variabilité.

Une grande partie de la variabilité, en particulier celle qui est observée au niveau intraclonal d'un site à l'autre, est probablement due à cette influence du milieu. Pour pallier cette difficulté et augmenter la précision expérimentale, il serait nécessaire de pratiquer un plus grand nombre de points d'inoculations sur un nombre plus large de ramets. Cette voie peut cependant paraître peu réaliste du fait des contraintes techniques qu'elle implique.

Une autre possibilité serait de mieux homogénéiser le diamètre des rameaux inoculés, la vigueur de ceux-ci semblant jouer un rôle important dans le déroulement de la maladie. L'importance de ce paramètre reste cependant à évaluer de façon plus précise.

Remerciements : Nous tenons à remercier B. Issenhuth, P. Legroux, C. Loustal, J.E. Ménard, M. Perochon et P. Simmer de l'Inra pour leur aide technique précieuse lors des inoculations et des lectures ainsi que les responsables de la Pépinière administrative de Peyrat-Le-Château pour l'accès au matériel végétal.

\section{RÉFÉRENCES}

[1] Baradat P., Desprez-Loustau M.L., Analyse diallèle et intégration de la sensibilité à la rouille courbeuse dans le programme d'amélioration du pin maritime, Ann. Sci. For. 54 (1997) 83-106.
[2] Bruck R.I., Manion, P.D., Interacting environmental factors associated with the incidence of Hypoxylon canker on trembling aspen, Can. J. For. Res. 10 (1980) 17-24.

[3] Bürgi A., Wachstum und Krebsanfälligkeit von Lärchenprovenienzen auf verschiedenen Standorten in der Schweiz, Forst und Holz 2 (1989) 28-33.

[4] Conradie E., Swart W.J., Wingfield M.J., Susceptibility of Eucalyptus grandis to Cryphonectria cubensis, Eur. J. For. Path. 22 (1992) 312-315.

[5] Dagnelie P., Théorie et méthodes statistiques I. Les méthodes de l'inférence statistique, Presses agronomiques de Gembloux, Gembloux, 1975.

[6] Hanson P., Susceptibility of different provenances of Pinus sylvestris, Pinus contorta and Picea abies to Gremmeniella abietina, Eur. J. For. Path. 1 (1997) 21-32.

[7] Hühn M., Beiträge zur Erfassung der phänotypischen Stabilität I. Vorschlag einiger auf Ranginformationen beruhenden Stabilitätsparameter, Medizin und Biologie 10 (4) (1979) 112-117.

[8] Jacques, D., Synthèse des résultats des tests de provenances de mélèze d'Europe en Belgique, Stat, Recherches forestières de Gembloux, Travaux Série E, nr 8 (1991) 39 pp.

[9] Knapp S.J., Stroup W.W., Ross W.M., Exact confidence intervals for heritability on a progeny mean basis, Crop Sci. 25 (1985) 192-194.

[10] Kojwang H.O., Variation and heritability of resistance of lodgepole pine to Wester gall rust, Eur. J. For. Path. 24 (1994) 137-143.

[11] Kulej M., Silvicultural value of various Polish provenances of larch in mountain conditions of experimental area in Krynica (en polonais), Sylwan, 11-12 (1989) 31-42.

[12] Muranty H., Santi F., Pâques L. E, Dufour J., Nombre optimal de ramets par clone dans deux tests clonaux, Ann. Sci. For. 53 (1996) 123-138.

[13] Nassar R., Hühn M., 1987. Studies on estimation of phenotypic stability : tests of significance for nonparametric measures of phenotypic stability, Biometrics 43 (1987) 45-53. 
[14] Pâques L.E., Inheritance and estimated genetic gains in a clonal test of hybrid larch (Larix $\times$ eurolepis), Scand. J. For. Res. 7 (1992) 355-365.

[15] Russell J.H., Libby W.J., Clonal testing efficiency : the trade-offs between clones tested and ramets per clone, Can. J. For. Res. 16 (1986) 925-930.

[16] Schober R., Neue Ergebnisse des II. Internationalen Lärchenprovenienzversuches von 1958/59 nach Aufnahmen von Teilversuchen in 11 europäischen Ländern und den USA. Schriften aus der Forstlichen Fakultät der Universität Göttingen und der Niedersächsischen Forstlichen Versuchsanstalt, Band 83 (1985) 164 pp.

[17] Swedjemark G., Stenlid J., Karlsson B., Genetic variation among clones of Picea abies in resistance to growth of Heterobasidium annosum, Silvae Genet. 46 (1997) 369-374.

[18] Sylvestre G., Delatour C., Réceptivité des blessures aux ascospores de Lachnellula willkommii (Hartig) Dennis chez Larix decidua Mill., Ann. Sci. For. 45 (1990) 57-66.
[19] Sylvestre G., Pâques L.E., Delatour C., Une méthode d'inoculation pour l'évaluation précoce du comportement du mélèze vis-à-vis du Lachnellula willkommii, Eur. J. For. Pathol. 24 (1994) 160-170.

[20] Sylvestre-Guinot G., Delatour C., Possibilités d'appréciation de la sensibilité du genre Larix au Lachnellula willkommii (Hartig) Dennis par inoculations artificielles, Ann. Sci. For., 40 (1983) 337-354.

[21] Yde-Andersen A., Host spectrum, host morphology and geographic distribution of larch canker, Lachnellula willkommii, Eur. J. For. Path. 9 (1979) 211-219.

[22] Zycha H., Bewertung von Lärchenkrebsbefall (Trichoscyphella willkommii) auf Versuchsflächen, Allg. Forstu. J.-Ztg. 149 (1978) 44-52.

[23] Zycha H., Lärchen- und Stammkrebsbefall, Allg. Forstu. J.-Ztg. 151 (1980) 112-117. 